\title{
Design and implementation of an on-line estimator for a rougher flotation process
}

\author{
ROLF HENRIKSEN $\dagger$ and THOR O. OLSEN $\ddagger$
}

Keywords: flotation processes, on-line estimation, non-linear filtering, identification, aggregation.

The problem of estimating grades and flotation parameters in a bank of flotation cells is investigated. From extensive simulation studies with both single-cell and multi-cell processes, an estimator based on an aggregate model of the bank is developed to estimate concentrate grades and aggregate flotation parameters. Specific results from the estimator's performance in a real flotation plant are reported.

\section{Introduction}

Flotation is a process widely used in the mining industries to separate from each other different minerals, e.g., chalcopyrite, sphalerite, gangue, etc., which are present in the ore. The process is based upon the fact that different minerals may have different physical (and chemical) surface properties. By systematically utilizing these properties, it is possible to separate the minerals from each other.

Flotation is usually carried out as outlined in Fig. 1. A mixture of ground ore and liquid is fed into a tank or vat. The liquid consists primarily of water, but some chemicals are added in order to make a group of the mineral particles aerophilic, i.e., affine to air bubbles. Air is blown through the vat from the bottom, and because of their affinity to air, some of the mineral particles will adhere to the air bubbles and rise to the top of the pulp, where a froth layer is formed. The froth, i.e. the concentrate, is then finally removed by a mechanical device in order to be dried or, eventually, to be sent to another flotation process for further refining.

Flotation may be carried out either as a batch process or continuously. In a continuous process the leftovers from one flotation cell, i.e., the so-called tailings are fed into another one and so on until most of the valuable minerals have been removed. It may also be the case, occasionally, that the invaluable minerals are removed whereas the valuable minerals are left in the tailings (so-called negative flotation).

The chemicals which are added to the pulp will actually make several groups of particles more or less affine to air, and because of varying particle sizes, even the particles of one mineral may have different affinity to air. The concentrate will therefore generally also contain unwanted minerals.

Many papers, books, and reports on modeling, simulation, and control of flotation processes have been published, see, e.g., Arbiter and Harris (1962), Fournier and Smith (1972), King (1975), Olsen (1975), Fuerstenau (1976), and Smith (1975). The most commonly used models today are based on the concept of so-called flotation classes, see, e.g., Olsen (1975). This concept originates from the assumption that there

Received 5 October 1983.

$\dot{\dagger}$ The Norwegian Institute of Technology, Division of Engineering Cybernetics.

$\ddagger$ COMPUTAS, Ingv. Ystgaards vei 15, N-7000 Trondheim, Norway. 
exists only a countable (usually finite) number of flotation rates, i.e., the material can be divided into groups or classes, flotation classes, where each class has a specific flotation rate which is different from the flotation rates of the other. This leads to fairly simple but, for most practical purposes, quite satisfactory models. It should be noted, however, that the assumption about a finite number of flotation classes usually is an approximation. The number of flotation classes will generally more or less resemble a continuum, which frequently can be approximated by a finite number. For more comments about this and alternative flotation models, see Olsen (1975).

The assumption about a finite number of flotation classes usually leads to time varying parameters in the model due to factors such as varying mineralogical properties, amount of liberation, and particle size distribution in the grinding process.
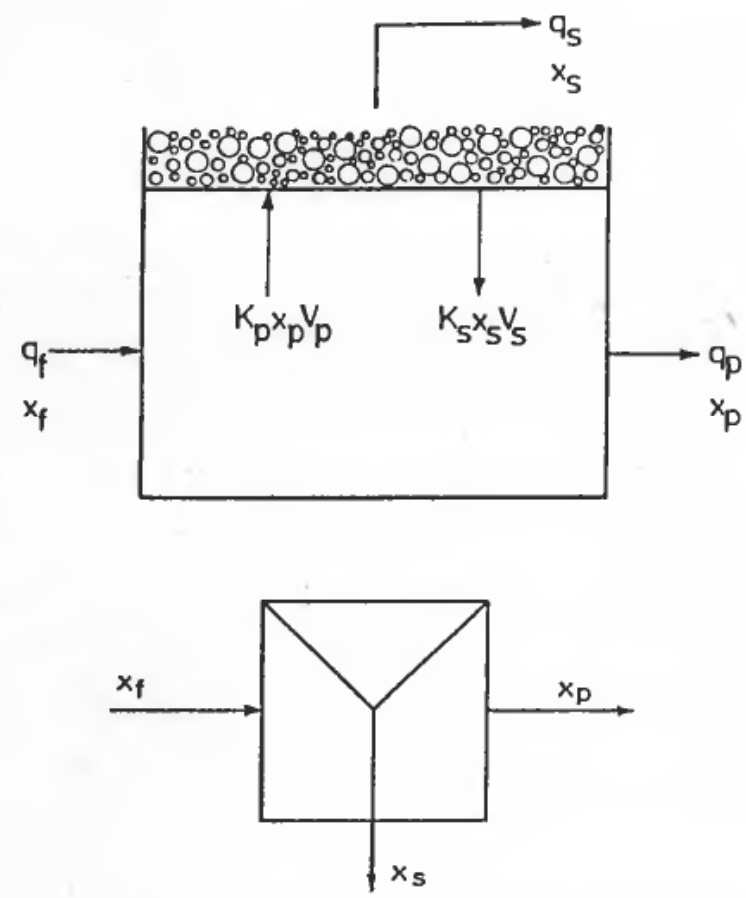

Figure 1. Schematic view of a flotation cell and a commonly used symbol for a flotation cell.

The rationale for developing an estimator for flotation plant was to synthesize a controller where the estimator was part of this controller. In order to find a suitable form of this estimator, comprehensive simulation studies were made to see which parameters actually could be estimated from normal operating data. These experiments were carried out both for single-cell and multi-cell processes, and the final form of the estimator was derived from these experiments.

The paper is organized as follows. In $\S 2$ we present the model for a single-cell process. Several estimators for single-cell processes are derived in $\S 3$ and tested through simulation studies. The model for multi-cell processes is derived in $\S 4$, and an estimator for such processes is developed in $\S 5$. Result from the implementation in the flotation plant at Folldal Verk, Norway, are presented in $\S 6$. 


\section{Modeling of single-cell processes}

We have in the previous section given a brief description of flotation processes. In this section we shall present a model of a single-cell process based upon the concept of flotation classes. A schematic view of a single-cell process is shown in Fig. 1. Assume the material in the pulp can be divided into $n$ flotation classes, and let $x_{p}, x_{s}$, and $x_{f}$ (all $n$-vectors) denote the grades (concentrations) of the different flotation classes in, respectively, the pulp (i.e., the tailings since perfect mixing is being assumed), the concentrate (i.e., the froth layer), and the feed. Furthermore, let $V^{\prime}{ }_{p}$ denote the pulp volume, $V^{\prime}{ }_{s}$ the froth volume, $q_{f}$ the feed flow rate, and $q_{p}$ the tailings flow rate. Finally, let $k_{p, J}$ and $k_{s, j}$, respectively, denote the flotation and drainage rate constants of the $j$ th flotation class. Defining

$$
k_{p}=\left(k_{p, 1}, \ldots, k_{p, n}\right)^{T}, \quad k_{s}=\left(k_{s, 1}, \ldots, k_{s, n}\right)^{T}, \quad K_{p}=\operatorname{diag}\left\{k_{p}\right\}, \quad K_{s}=\operatorname{diag}\left\{k_{s}\right\}
$$

we obtain, by considering the mass balances in the cell, the following model described by Itô stochastic differential equations

$$
\begin{aligned}
& d x_{p}(t)=-\left(K_{p}+\frac{\gamma}{\tau_{f}} I\right) x_{p}(t) d t+\alpha K_{s} x_{s}(t) d t+\frac{1}{\tau_{f}} x_{f}(t) d t+d \beta_{p}(t) \\
& d x_{s}(t)=\frac{1}{\alpha} K_{p} x_{p}(t)-\left(K_{s}+\frac{1-\gamma}{\alpha \tau_{f}} I\right) x_{s}(t) d t+d \beta_{s}(t)
\end{aligned}
$$

where

$$
\gamma=\frac{q_{p}}{q_{f}}, \quad \tau_{f}=\frac{V_{p}^{\prime}}{q_{f}}, \quad \alpha=\frac{V_{s}^{\prime}}{V_{p}^{\prime}}
$$

and where $\left\{\beta_{p}(t)\right\}$ and $\left\{\beta_{s}(t)\right\}$ are (zero-mean) Wiener processes with covariance parameter matrices $V_{p}(t)$ and $V_{s}(t)$, respectively.

The feed grade vector $x_{f}(t)$ is assumed to be a stochastic process with independent increments and slowly varying, i.e., approximately constant mean value function $\bar{x}_{f}(t)$. The model of $x_{f}(t)$ then takes the form

$$
d x_{f}(t)=d \beta_{f}(t)
$$

where $\left\{\beta_{f}(t)\right\}$ is a (zero-mean) Wiener process with known covariance parameter matrix $V_{f}(t)$.

Due to factors such as varying mineralogical properties, amount of liberation, and particle size distribution in the grinding process, both flotation and drainage rate constants will be time varying. Although it is generally acknowledged that these parameters depend, among others, on $x_{p}$ and $x_{s}$, viz., $k_{p}=k_{p}\left(x_{p}, x_{s}\right)$ and $k_{s}=k_{s}\left(x_{p}, x_{s}\right)$, we shall assume all these parameters to be slowly varying with independent increments. The model for these parameters thus becomes

$$
d k_{p}(t)=d \beta_{k p}(t), \quad d k_{s}(t)=d \beta_{k s}(t)
$$

where $\left\{\beta_{k p}(t)\right\}$ and $\left\{\beta_{k s}(t)\right\}$ are (zero-mean) Wiener processes with covariance parameter matrices $V_{k p}(t)$ and $V_{k s}(t)$, respectively.

In the actual flotation plant which is investigated, we can assume the parameters $\tau_{f}$ and $\alpha$ to be constants because the relative changes in these parameters are very small. Also, the relative changes in the parameter $\gamma$ are very small, but this parameter is very close to I (see Fig. 7), so that the relative changes in $1-\gamma$, which enters Eqn. (3), may 
be quite large. We shall therefore consider the parameter $\gamma$ to be time varying, and the model for it is taken to be

$$
d \gamma(t)=d \beta_{\gamma}(t)
$$

where $\left\{\beta_{\gamma}(t)\right\}$ is a (zero-mean) Wiener process with variance parameter $V(t)$.

We are now ready to define the augmented state $(x)$ and plant noise $(\beta)$ vectors for the cell

$$
x^{T}=\left(x_{p}{ }^{T}, x_{s}{ }^{T}, x_{f}{ }^{T}, k_{p}{ }^{T}, k_{s}{ }^{T}, \gamma\right), \quad \beta=\left(\beta_{p}{ }^{T}, \beta_{s}{ }^{T}, \beta_{f}{ }^{T}, \beta_{k p}{ }^{T}, \beta_{k s}{ }^{T}, \beta_{\gamma}\right)
$$

where the dimensions of both of the vectors are equal to $5 n+1$. The model, consisting of Eqns. (2)-(6), can now be written in the compact form

$$
d x(t)=f(x(t)) d t+d \beta(t)
$$

where the definition of the vector function $f$ is obvious from the context.

Two sets of measurements are being carried out. The first set consists simply of measurements of the feed and tailings flow rates, and they are carried out at a fairly high sampling rate. By a simple transformation we can consider this to be a scalar measurement of the form

$$
y_{1}\left(t_{k}\right)=q_{f}\left(1-\gamma\left(t_{k}\right)\right)+w_{1}\left(t_{k}\right)
$$

where $\left\{w_{1}\left(t_{k}\right)\right\}$ is a zero-mean white Gaussian sequence with variance $W_{1}\left(t_{k}\right)$.

The second set consists of measurements of the grades in the feed, tailings, and, which at the design stage was believed, the concentrate (it later turned out that measurements of concentrate grades were not available). The model for these measurements takes the form

$$
\begin{aligned}
& y_{2}\left(t_{i}\right)=H x_{p}\left(t_{i}\right)+w_{2}\left(t_{i}\right) \\
& y_{3}\left(t_{j}\right)=H x_{f}\left(t_{j}\right)+w_{3}\left(t_{j}\right) \\
& y_{4}\left(t_{l}\right)=H x_{s}\left(t_{l}\right)+w_{4}\left(t_{l}\right)
\end{aligned}
$$

where $\left\{w_{2}\left(t_{i}\right)\right\},\left\{w_{3}\left(t_{j}\right)\right\}$, and $\left\{w_{4}\left(t_{l}\right)\right\}$ are zero-mean Gaussian sequences with covariance matrices $W_{2}\left(t_{i}\right), W_{3}\left(t_{j}\right)$, and $W_{4}\left(t_{l}\right)$, respectively. All these measurements are carried out at a low sampling rate. None of the measurements, including $y_{1}$, are generally carried out simultaneously.

By subtracting $q_{f}$ from $y_{1}$ in Eqn. (9), we can rewrite this equation as

$$
y_{1}\left(t_{k}\right)=-q_{f} \gamma\left(t_{k}\right)+w_{1}\left(t_{k}\right)
$$

We can then, in an obvious manner, rewrite Eqns. (10)-(13) as

$$
y\left(t_{k}\right)=D\left(t_{k}\right) x\left(t_{k}\right)+w\left(t_{k}\right)
$$

where $y\left(t_{k}\right), D\left(t_{k}\right)$, and $w\left(t_{k}\right)$ depend on which measurement is being carried out, e.g., $y\left(t_{k}\right)=y_{1}\left(t_{k}\right), w\left(t_{k}\right)=w_{1}\left(t_{k}\right)$, and $D\left(t_{k}\right)=\left[0,0,0,0,0,-q_{f}\right]$ if the measurement is of the type given by Eqn. (9).

\section{Design of an estimator for a single-cell process}

At the first stage of the design procedure we were quite uncertain about how good estimates of the unknown parameters could actually be obtained. The following 
methods were investigated through extensive simulation experiments (Olsen and Henriksen, 1976, 1977):

Extended Kalman filter

Second-order non-linear filter

Maximum likelihood method

The results from these simulation experiments are reviewed in the sequel.

\section{Extended Kalman filter}

The form of the extended Kalman filter is given in Jazwinski (1970). Let $\hat{x}\left(t \mid t_{k}\right)$ denote the estimate of the augmented state vector $x(t)$ at time $t>t_{k}$ given the set of observations $Y\left(t_{k}\right)=\left\{y\left(t_{0}\right), y\left(t_{1}\right), \ldots, y\left(t_{k}\right)\right\}$ where $y\left(t_{i}\right)$ is either $y_{1}\left(t_{i}\right), y_{2}\left(t_{i}\right), y_{3}\left(t_{i}\right)$, or $y_{4}\left(t_{i}\right)$. The predictor between observations takes the form

$$
\begin{aligned}
& \dot{\hat{x}}\left(t \mid t_{k}\right)=f\left(\hat{x}\left(t \mid t_{k}\right)\right) \\
& \dot{P}\left(t \mid t_{k}\right)=\hat{A} P\left(t \mid t_{k}\right)+P\left(t \mid t_{k}\right) \hat{A}^{T}+V(t)
\end{aligned}
$$

where $P\left(t \mid t_{k}\right)$ is the covariance matrix of $x(t)$ given $Y\left(t_{k}\right)$. $V(t)$ is the covariance matrix of the Wiener process $\{\beta(t)\}$, whereas $\hat{A}$ is the matrix of partial derivatives of $f(f$ being defined by Eqns. (2)-(8)) with respect to $x$ at $x=\hat{x}\left(t \mid t_{k}\right)$, viz.

$$
\hat{A}=\frac{\partial f}{\partial x^{T}}\left(\hat{x}\left(t \mid t_{k}\right)\right)
$$

The filtered estimates are given from

where

$$
\begin{aligned}
& \hat{x}\left(t_{k+1} \mid t_{k+1}\right)=\hat{x}\left(t_{k+1} \mid t_{k}\right)+K\left(t_{k+1}\right)\left[y\left(t_{k+1}\right)-\hat{y}\left(t_{k+1} \mid t_{k}\right)\right] \\
& P\left(t_{k+1} \mid t_{k+1}\right)=\left[I-K\left(t_{k+1}\right) D\left(t_{k+1}\right)\right] P\left(t_{k+1} \mid t_{k}\right)
\end{aligned}
$$

$$
\begin{aligned}
\hat{y}\left(t_{k+1} \mid t_{k}\right) & =D\left(t_{k+1}\right) \hat{x}\left(t_{k+1} \mid t_{k}\right) \\
K\left(t_{k+1}\right) & =P\left(t_{k+1} \mid t_{k}\right) D^{T}\left(t_{k+1}\right)\left[D\left(t_{k+1}\right) P\left(t_{k+1} \mid t_{k}\right) D^{T}\left(t_{k+1}\right)+W\left(t_{k+1}\right)\right]^{-1}
\end{aligned}
$$

and where $W\left(t_{k+1}\right)=W_{1}\left(t_{k+1}\right)$ if $y\left(t_{k+1}\right)=y_{1}\left(t_{k+1}\right), W\left(t_{k+1}\right)=W_{2}\left(t_{k+1}\right)$ if $y\left(t_{k+1}\right)=$ $y_{2}\left(t_{k+1}\right)$, and so on.

Typical samples from the simulation experiments with the foregoing estimator are shown in Figs. 2 and 3. The estimates of the grades in both the feed, the tailings, and the concentrate are quite good, but this is not surprising since they are all assumed to be measured. Also, the estimate of the parameter $\gamma$ is extremely good. However, the estimates of both the flotation rate constant $\left(k_{p, 1}\right)$ and the drainage rate constant $\left(k_{s, 1}\right)$ are quite bad, and these parameters are obviously quite poorly observable. This is caused by the fact that a linear combination of $k_{p, i}$ and $k_{s, i}, i=1, \ldots, n$, expresses the net flow of material from the pulp to the froth layer, and only the net flow of material can in a reasonable manner be estimated from the available observations.

The so-called net flotation rate constants express the net flow of material to the froth layer and are related to the other variables by the expression

$$
N_{i}=\frac{k_{p, i} x_{p, i}-\alpha k_{s, i} x_{s, i}}{x_{p, i}}, \quad i=1, \ldots, n
$$

and these parameters can be quite accurately estimated as seen in Fig. $3(d)$. We have in this figure also shown the value of $\hat{k}_{p, 1}$ with $\hat{k}_{s, 1}=0$ in the estimator, and it is seen 
(a)

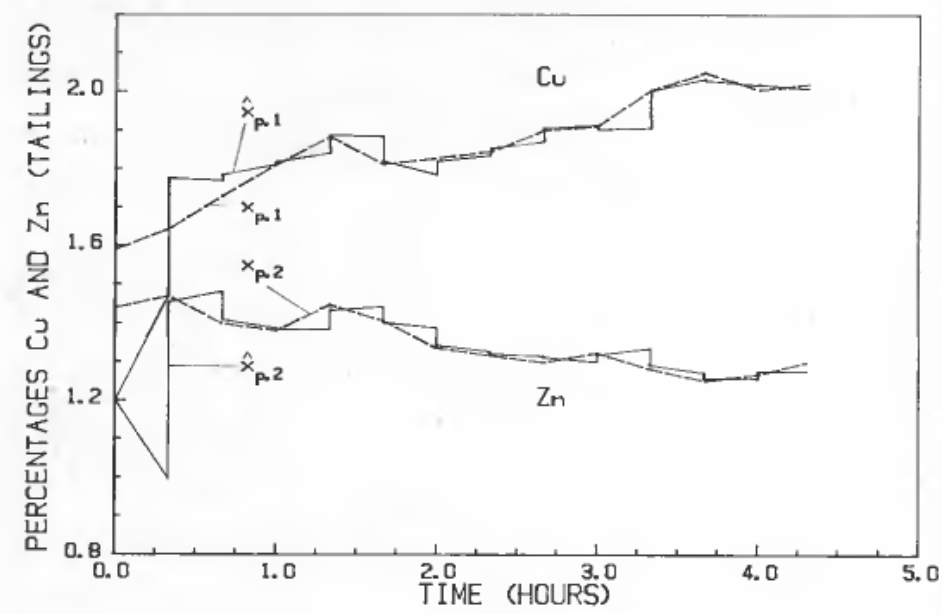

(b)

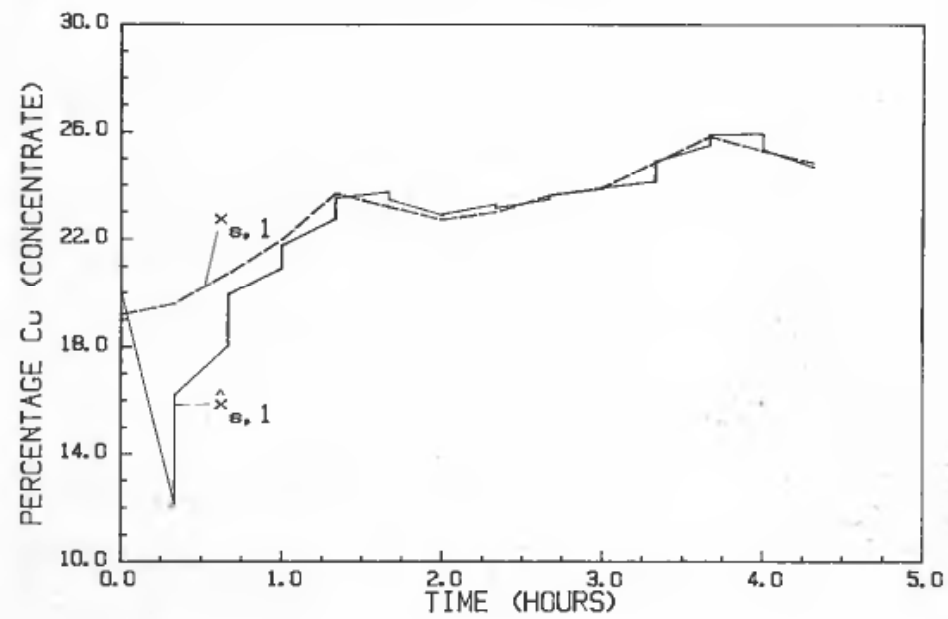

Figure 2. Simulated and estimated grades (by volume) of a single-cell process.

to be very close to $\hat{N}_{1}$ (in fact, from Eqn. (22), $\hat{N}_{i}=\hat{k}_{p, i}$ if $\hat{k}_{s, i}=0$ ). Eqn. (22) expresses the linear combination of $k_{p, i}$ and $k_{s, i}$ which is properly observable, viz.

$$
k_{p, i} x_{p, i}-\alpha k_{s, i} x_{s, i}=N_{i} x_{p, i}
$$

where $k_{p, i}=N_{i}$ and $k_{s, i}=0$ is one of the possible solutions.

According to the simulation experiments it was apparent that only net flotation rate constants could be properly estimated with this type of estimator.

\section{Second-order non-linear filter}

There exists actually two forms of this filter, the truncated second-order non-linear filter and the Gaussian second-order non-linear filter, see Jazwinski (1970). Although some apparent discrepancies between these two filters have existed (this has recently been solved by Henriksen, 1982), they turn out to be identical for the type of process models (second-order non-linearities in the state equations and linear observation equations) which is being used in this paper. 

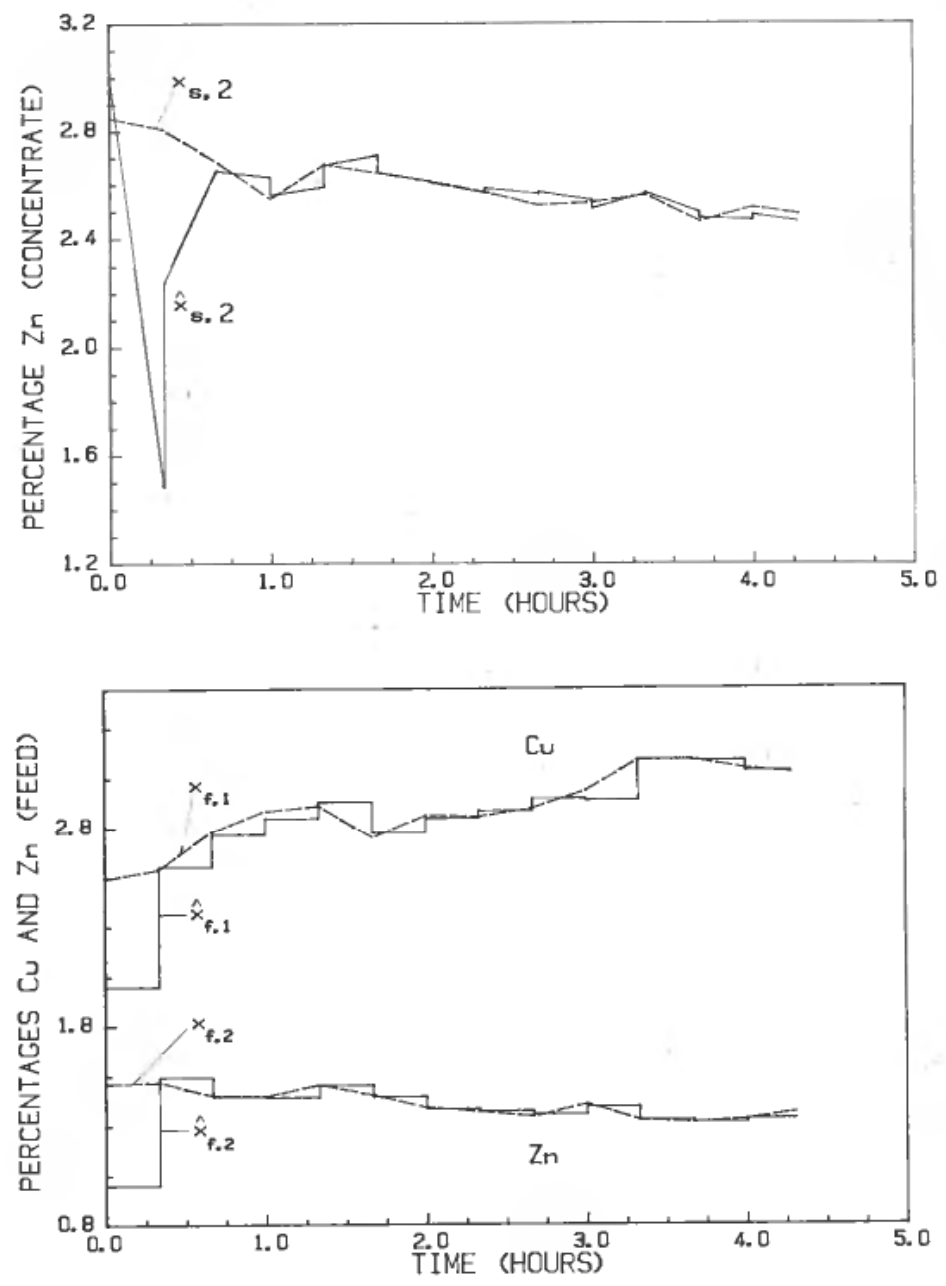

(d)

Figure 2-continued.

The rationale for trying out a second-order non-linear filter was the fact that all non-linearities was of second order in the process model. If all probability distributions could be assumed to be fairly symmetrical, a near-optimal non-linear filter for the system could be developed because third order central moments, which appear in the covariance equation of the optimal filter for such models would drop out.

The only difference from the preceding filter turns out to appear in Eqn. (15), which now has to be replaced by

$$
\dot{\hat{x}}\left(t \mid t_{k}\right)=f\left(\hat{x}\left(t \mid t_{k}\right)\right)+\frac{1}{2} f_{x x}\left(\hat{x}\left(t \mid t_{k}\right)\right)\left[\operatorname{cs} P\left(t \mid t_{k}\right)\right]
$$

where $f_{x x}=\partial / \partial x^{T} \cdot\left(\partial f / \partial x^{T}\right)$ and $c s$ denotes the column string operator, see Vetter $(1970,1973)$.

The performance of the second-order non-linear filter turned out to be almost identical to the extended Kalman filter, i.e., most of the results were not discernible. The foregoing figures could therefore also very well be the results from a simulation experiment with the second-order non-linear filter. 
(a)

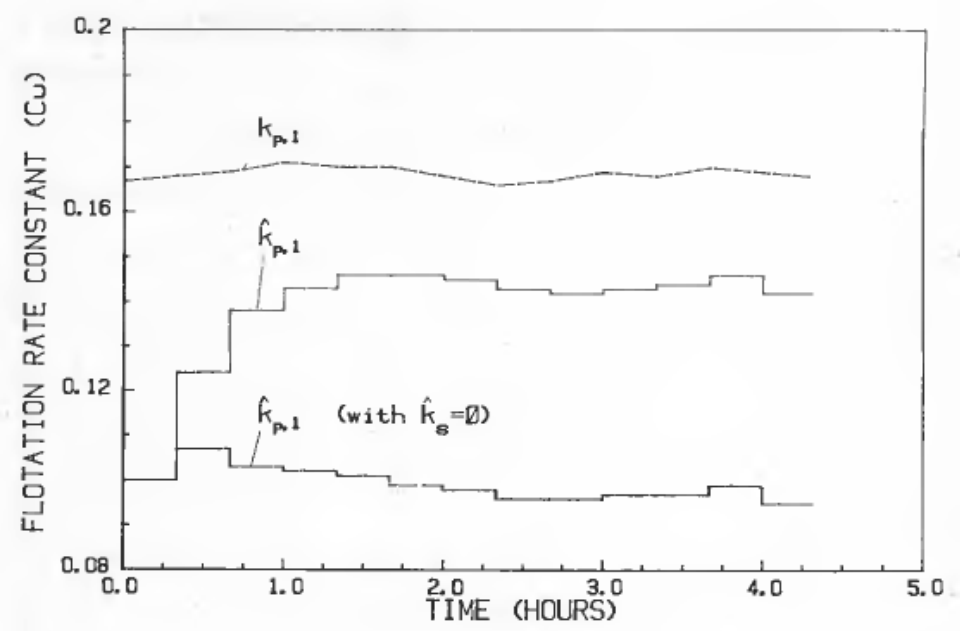

(b)

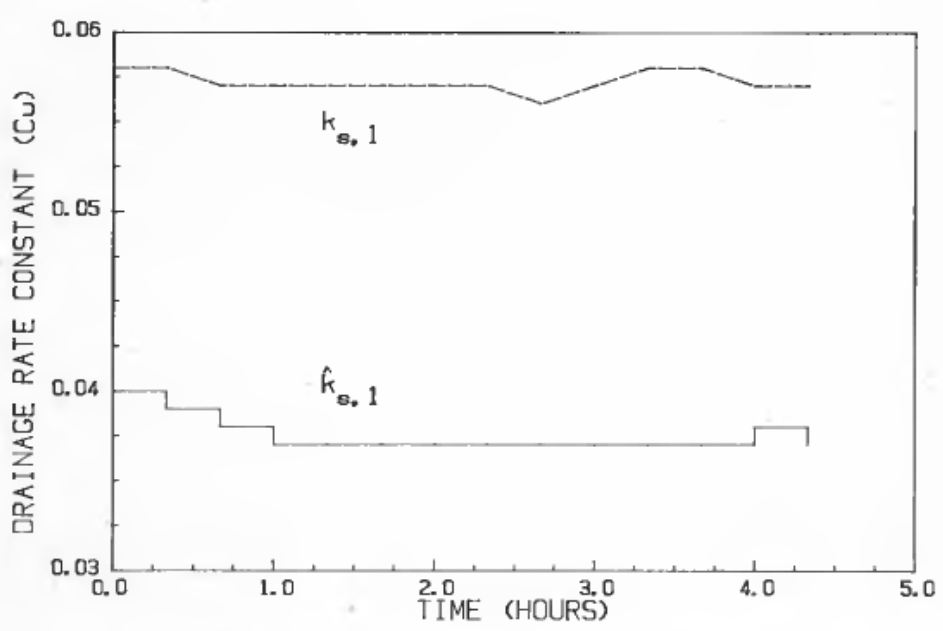

Figure 3. Simulated and estimated flotation parameters of a single-cell process.

\section{Maximum likelihood method}

The simulation experiments showed that both of the preceding recursive estimators were unable to estimate the flotation and drainage rate constants. In a paper by Hallingstad and Sælid (1976) it was reported that the maximum likelihood method (ML-method) yielded far better estimates of certain variables than the extended Kalman filter. It was therefore decided to investigate the performance of the MLmethod on flotation processes.

The ML-method, which is basically an off-line method (although approximative recursive ML-methods have been developed), has certain limitations when applied to flotation processes. The result of an ML-estimator is a parameter vector which minimizes the likelihood criterion on a set of past observations. When the parameters are time varying, as they ceftainly are in flotation processes, ML-estimates will have to be computed for relatively short time periods. Periods of four hours are long in this connection, and are considered to be an upper limit in the present application. On the other hand, the X-ray analyser is quite slow with a sampling interval of approximately 


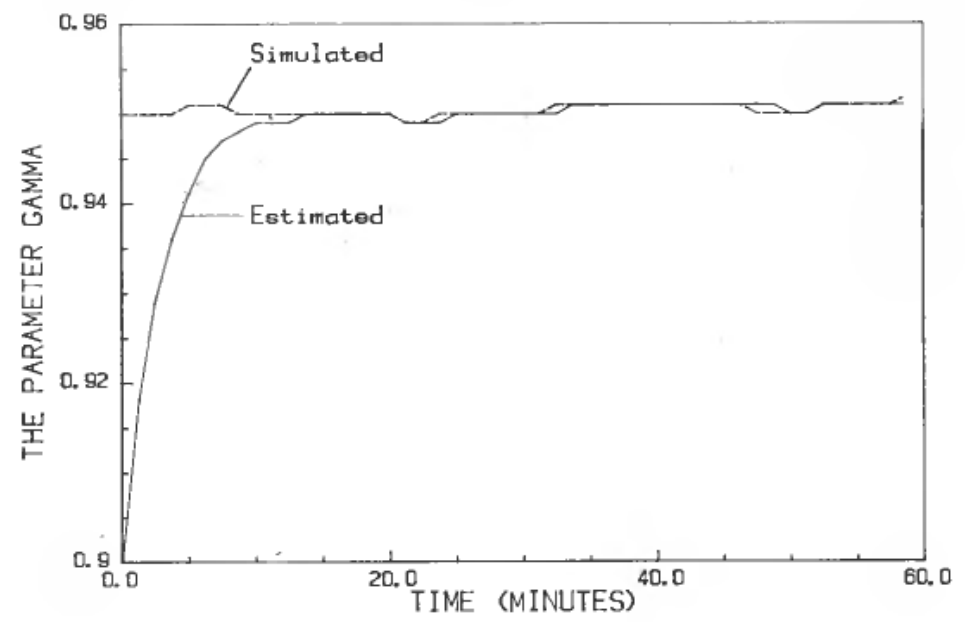

(c)

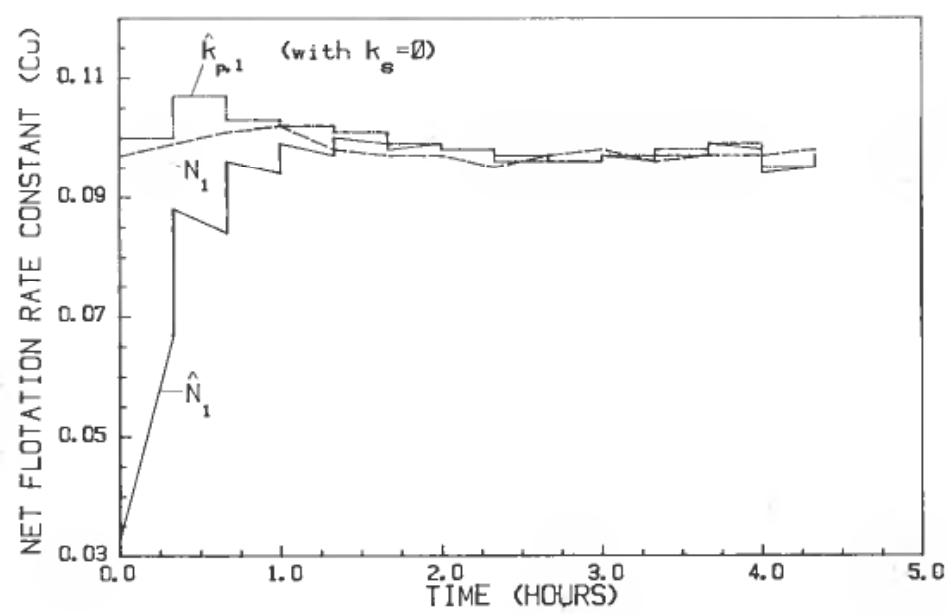

(d)

Figure 3-continued.

$20 \mathrm{~min}$. There are, therefore, only a few samples available for the ML-estimation scheme.

The use of ML-estimation in dynamic systems was developed many years ago by Kashyap (1970). The unknown parameter vector is assumed to be $\theta=\left(k_{p}{ }^{T}, k_{s}{ }^{T}\right)$, whereas the parameter $\gamma$ is assumed to be known. This can be justified from the simulation experiments (Fig. $3(c)$ ) which actually reveal that we can consider $\hat{\gamma}$ to be equal to $\gamma$ in this connection. The model now takes the following linear form (Eqns. (2)-(4) and (14))

$$
\begin{gathered}
d x(t)=A(\theta) x(t) d t+d \beta(t) \\
y\left(t_{k}\right)=D\left(t_{k}\right) x\left(t_{k}\right)+w\left(t_{k}\right)
\end{gathered}
$$

where $y\left(t_{k}\right)$ now is either $y_{2}\left(t_{k}\right), y_{3}\left(t_{k}\right)$, or $y_{4}\left(t_{k}\right)$. Note that the definitions of $x(t)$ and $\beta(t)$ are different in this case since parameters are not included in the state vector. Some of the elements in the matrix $A(\theta)$ are linear functions of the unknown parameters. 
In the papers by Kashyap (1970) or Mehra (1971), it is shown that the maximum likelihood estimate of $\theta$ given by

$$
\hat{\theta}=\operatorname{Arg}\left(\underset{\theta}{\operatorname{Max}} p\left(y\left(t_{0}\right), \ldots, y\left(t_{N}\right) \mid \theta\right)\right)
$$

is found by minimizing the likelihood functional

$$
J=\frac{1}{2} \sum_{k=0}^{N}\left[\epsilon^{T}\left(t_{k}\right) R^{-1}\left(t_{k}\right) \epsilon\left(t_{k}\right)+\log \left(\operatorname{det} R\left(t_{k}\right)\right)\right]
$$

where $\left\{\epsilon\left(t_{k}\right), k=0,1, \ldots\right\}$ is the innovation process, viz.

$$
\epsilon\left(t_{k}\right)=y\left(t_{k}\right)-\hat{y}\left(t_{k} \mid t_{k-1}, \theta\right)=y\left(t_{k}\right)-D\left(t_{k}\right) \hat{x}\left(t_{k} \mid t_{k-1}, \theta\right)
$$

for a given parameter vector $\theta$, whereas $R\left(t_{k}\right)$ is the covariance matrix of the innovation process, viz.

$$
R\left(t_{k}\right)=E\left\{\epsilon\left(t_{k}\right) \epsilon^{T}\left(t_{k}\right) \mid t_{k-1}, \theta\right\}
$$

$\epsilon\left(t_{k}\right)$ and $R\left(t_{k}\right)$ are computed from the following set of equations. The predictor between observations has the form

$$
\begin{aligned}
& \dot{\hat{x}}\left(t \mid t_{k}, \theta\right)=A(\theta) \hat{x}\left(t \mid t_{k}, \theta\right), \quad t>t_{k} \\
& \dot{P}\left(t \mid t_{k}, \theta\right)=A(\theta) P\left(t \mid t_{k}, \theta\right)+P\left(t \mid t_{k}, \theta\right) A^{T}(\theta)+V(t), \quad t>t_{k}
\end{aligned}
$$

where $V(t)$ has been changed according to the new definition of $\beta(t)$.

Finally,

$$
\begin{aligned}
& \hat{x}\left(t_{k+1} \mid t_{k+1}, \theta\right)=\hat{x}\left(t_{k+1} \mid t_{k}, \theta\right)+K\left(t_{k+1}\right) \epsilon\left(t_{k+1}\right) \\
& P\left(t_{k+1} \mid t_{k+1}, \theta\right)=\left[I-K\left(t_{k+1}\right) D\left(t_{k+1}\right)\right] P\left(t_{k+1} \mid t_{k}, \theta\right)
\end{aligned}
$$

where

$$
\begin{aligned}
& K\left(t_{k+1}\right)=P\left(t_{k+1} \mid t_{k}, \theta\right) D^{T}\left(t_{k+1}\right) R^{-1}\left(t_{k+1}\right) \\
& R\left(t_{k+1}\right)=D\left(t_{k+1}\right) P\left(t_{k+1} \mid t_{k}, \theta\right) D^{T}\left(t_{k+1}\right)+W\left(t_{k+1}\right)
\end{aligned}
$$

Simulation experiments with the ML-estimator turned out, however, to be quite disappointing, see Olsen and Henriksen (1977). There was no change in this estimator's ability to estimate the flotation parameters compared to the two previous ones, and Figs. $3(a), 3(b)$, and $3(d)$ are quite representative also for the ML-estimation.

The conclusion that can be drawn from these experiments is that the grades in both the feed, the tailing, and the concentrate can be quite accurately estimated with on-line estimators, whereas only net flotation rate constants can be properly estimated with any of the three estimators that were tested. Naturally, the extended Kalman filter, which is the simplest one, was selected for further simulation experiments with multicell processes.

\section{Modeling of multi-cell processes}

Most flotation processes actually consist of a bank of flotation cells where the tailings from one cell is fed into the next one and so on, see Fig. 4. The model for such a process is based on the model which was developed for a single-cell process. Let 


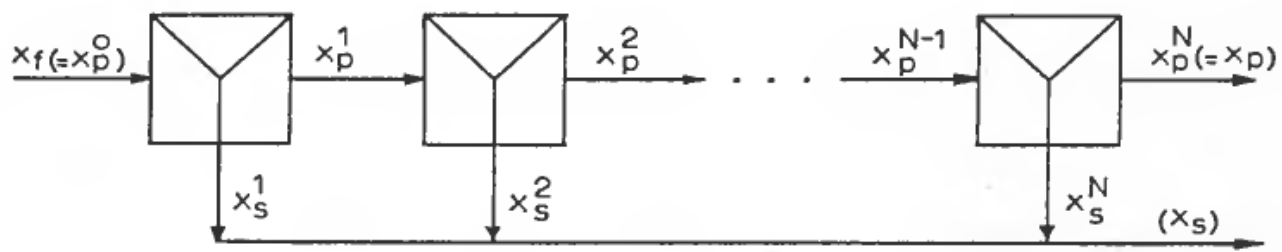

Figure 4. Multi-cell flotation process.

superscript $i$, e.g., $x_{p}{ }^{i}$, denote a variable in the $i$ th flotation cell. Using the same kind of notations as in the single-cell model, we obtain

$$
\begin{aligned}
\begin{aligned}
d x_{p}{ }^{i}(t)= & -\left(K_{p}{ }^{i}+\frac{\gamma^{i}}{\tau_{f}{ }^{i}} I\right) x_{p}{ }^{i}(t) d t+\alpha^{i} K_{s}{ }^{i} x_{s}{ }^{i}(t) d t \\
& +\frac{1}{\tau_{f}{ }^{i}} x_{p}{ }^{i-1}(t) d t+d \beta^{i}(t), \quad i=1, \ldots, N
\end{aligned} \\
d x_{s}^{i}(t)=\frac{1}{\alpha^{i}} K_{p}^{i} x_{p}{ }^{i}(t) d t-\left(K_{s}^{i}+\frac{1-\gamma^{i}}{\alpha^{i} \tau_{f}^{i}} I\right) x_{s}^{i}(t) d t+d \beta_{s}{ }^{i}(t), \quad i=1, \ldots, N
\end{aligned}
$$

where $N$ denotes the total number of flotation cells. Furthermore, we have defined $x_{p}{ }^{0}=x_{f}$. The remainder of the model takes the form (see Eqns. (5)-(6))

$$
\begin{aligned}
d k_{p}{ }^{i}(t) & =d \beta_{k p}{ }^{i}(t), \quad d x_{s}^{i}(t)=d \beta_{k s}{ }^{i}(t) \\
d \gamma^{i}(t) & =d \beta_{\gamma}{ }^{i}(t)
\end{aligned}
$$

where $\gamma^{i}, \tau_{f}^{i}$, and $\alpha^{i}$ are defined in the same way as in the single-cell model (e.g., $\left.\gamma^{i}=q_{p}{ }^{i} / q_{p}{ }^{i-1}\right)$.

In addition to the equations above we have to add the equation

$$
d x_{p}{ }^{0}(t)=d \beta_{f}(t)
$$

which is identical to Eqn. (4) since $x_{p}{ }^{0}=x_{f}$.

Eqns. (37)-(40) can compactly be written in the form

$$
d x^{i}(t)=f^{i}\left(x^{i}(t)\right) d t+\frac{1}{\tau_{f}^{i}} x^{i-1}(t) d t+d \beta^{i}(t), \quad i=1, \ldots, N
$$

where the definitions of $x^{i}, f^{i}$, and $\beta^{i}$ should be obvious from the context, e.g., the vector $x^{i}$ contains the subvectors $x_{p}{ }^{i}, x_{s}{ }^{i}, k_{p}{ }^{i}, k_{s}{ }^{i}$, and $\gamma^{i}$. The total number of states is equal to $(4 n+1) N+n$.

It was originally assumed that measurements of the feed and tailings flow rates, and of the feed, concentrate, and tailings grades could be carried out. The observation equations then take the form

$$
\begin{aligned}
& y_{1}\left(t_{k}\right)=-q_{f} \prod_{i=1}^{N} \gamma^{i}\left(t_{k}\right)+w_{1}\left(t_{k}\right) \\
& y_{2}\left(t_{i}\right)=H x_{p}^{0}\left(t_{i}\right)+w_{2}\left(t_{i}\right) \\
& y_{3}\left(t_{j}\right)=H\left[\frac{1}{\left(1-\prod_{i=1}^{N} \beta^{i}\left(t_{j}\right)\right)} \sum_{i=1}^{N}\left(x_{s}^{i}\left(t_{j}\right)\left(1-\beta^{i}\left(t_{j}\right)\right) \prod_{k=1}^{i-1} \beta^{k}\left(t_{j}\right)\right)\right]+w_{3}\left(t_{j}\right) \\
& y_{4}\left(t_{l}\right)=H x_{p}^{N}\left(t_{l}\right)+w_{4}\left(t_{l}\right)
\end{aligned}
$$


(a)

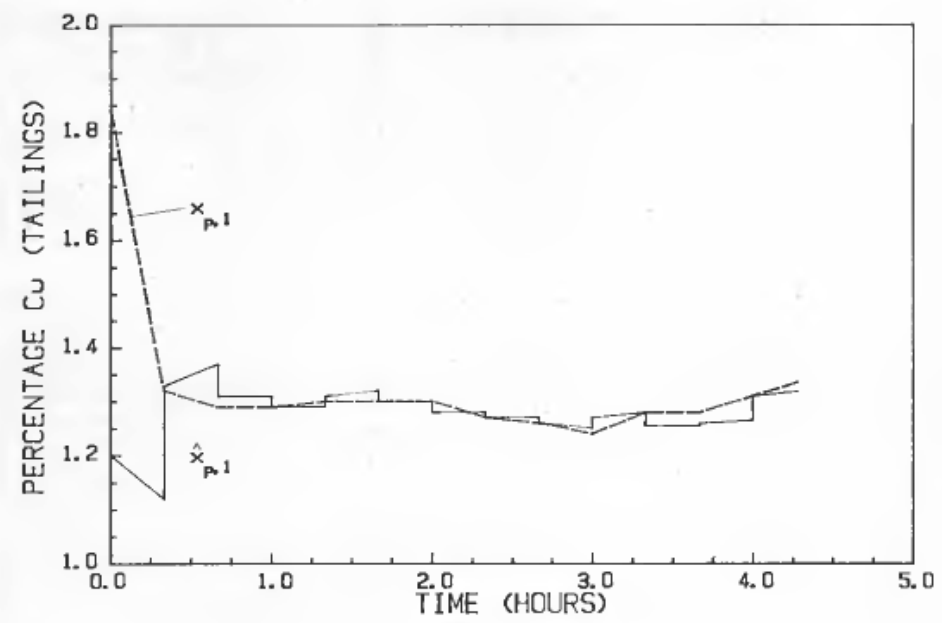

(b)

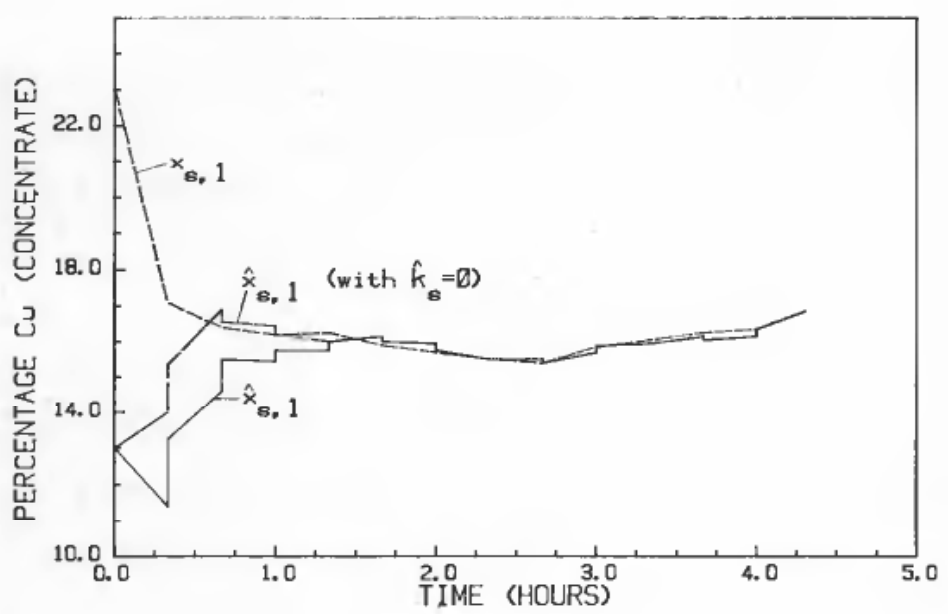

Figure 5. Simulated and estimated aggregate grades (by volume) and parameters of a multicell process.

\section{Design of an estimator for a multi-cell process based on an aggregate model}

From the simulation experiments it was obvious that the drainage rates could be dropped in the estimator's model. Furthermore, since no measurements were made of neither the feed, the tailings, nor the concentrate of each cell in the multi-cell process, it became apparent that the internal state of each cell could not be estimated from measurements of the feed, the tailings and the aggregate concentrate of the whole bank. It was therefore decided to use a single-cell aggregated model of the process in the design of an estimator for a multi-cell process, see Olsen and Henriksen (1981).

The aggregate model is identical to the model which was developed in $\S 2$. The relationships between the variables in the aggregate single-cell model and the variables in the multi-cell model are as follows

$$
q_{p}=q_{p}{ }^{N}, \quad q_{f}=q_{p}^{0}, \quad V_{p}=\sum_{i=1}^{N} V_{p}^{i}, \quad V_{s}=\sum_{i=1}^{N} V_{s}^{i}, \quad x_{p} \leftrightarrow x_{p}^{N}
$$




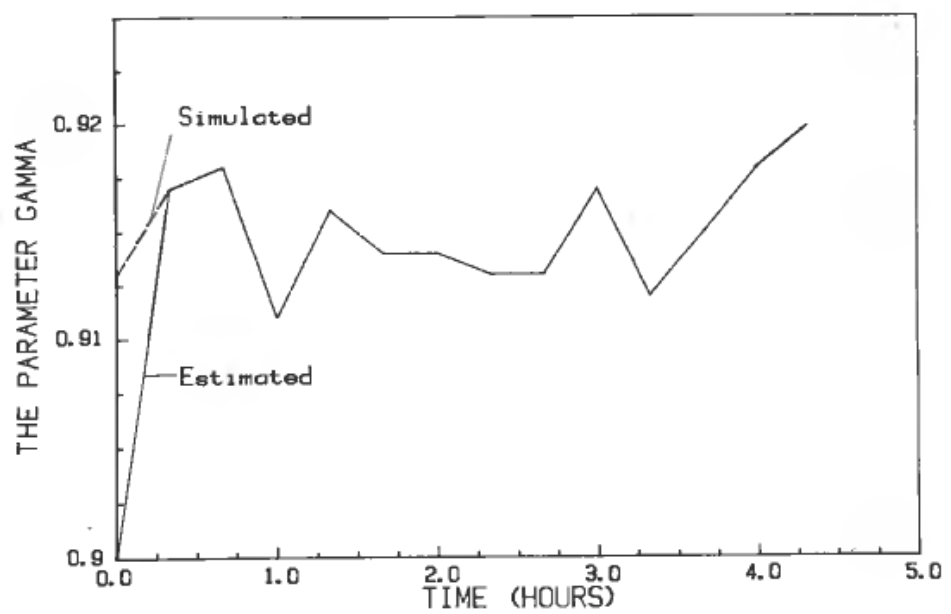

(c)

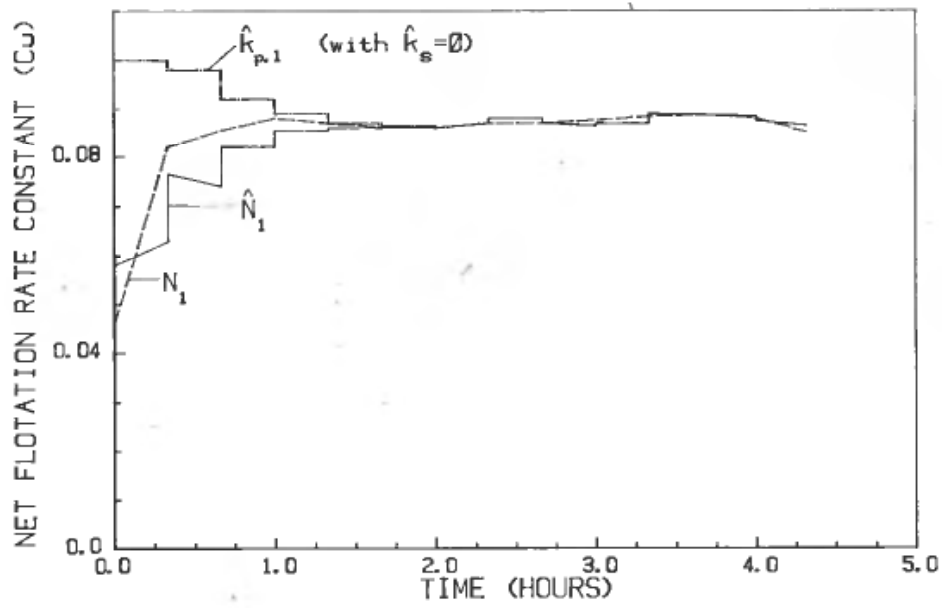

(d)

Figure 5-continued.

$$
\begin{aligned}
x_{s} \leftrightarrow \tilde{x}_{s} & =\frac{1}{\left(1-\prod_{=1}^{N} \gamma^{j}\right)} \sum_{i=1}^{N} x_{s}{ }^{i}\left(1-\gamma^{i}\right) \prod_{=1}^{i-1} \gamma^{j} \\
x_{f} \leftrightarrow x_{f} & =x_{p}^{0} \\
\gamma \leftrightarrow \tilde{\gamma} & =\prod_{i=1}^{N} \gamma^{i}=\frac{q_{p}{ }^{N}}{q_{p}{ }^{0}} \\
k_{p, j} \leftrightarrow \tilde{k}_{p, j} & =\frac{1}{x_{p, j} \sum_{i=1}^{N} V_{p}^{i}} \sum_{i=1}^{N} V_{p}^{i} k_{p, j} x_{p, j}, \text { flotation class } j=1, \ldots, n \\
k_{s, j} \leftrightarrow \tilde{k}_{s, j} & =\frac{1}{\tilde{x}_{s, j} \sum_{i=1}^{N} V_{s}^{i}} \sum_{i=1}^{N} V_{s}^{i} k_{s, j}{ }^{i} x_{s, j}{ }^{i}, \quad \text { flotation class } j=1, \ldots, n
\end{aligned}
$$


(a)

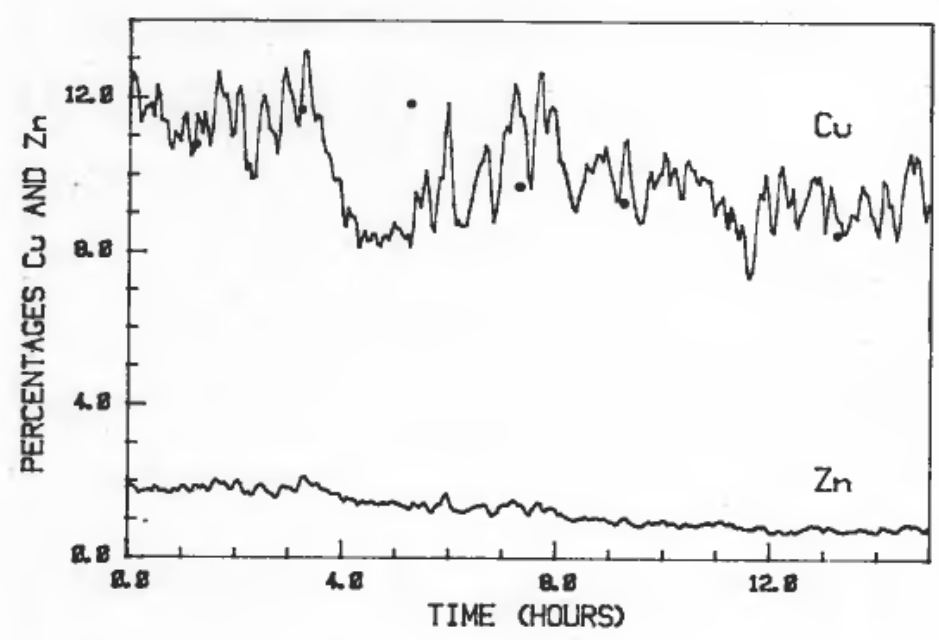

(b)

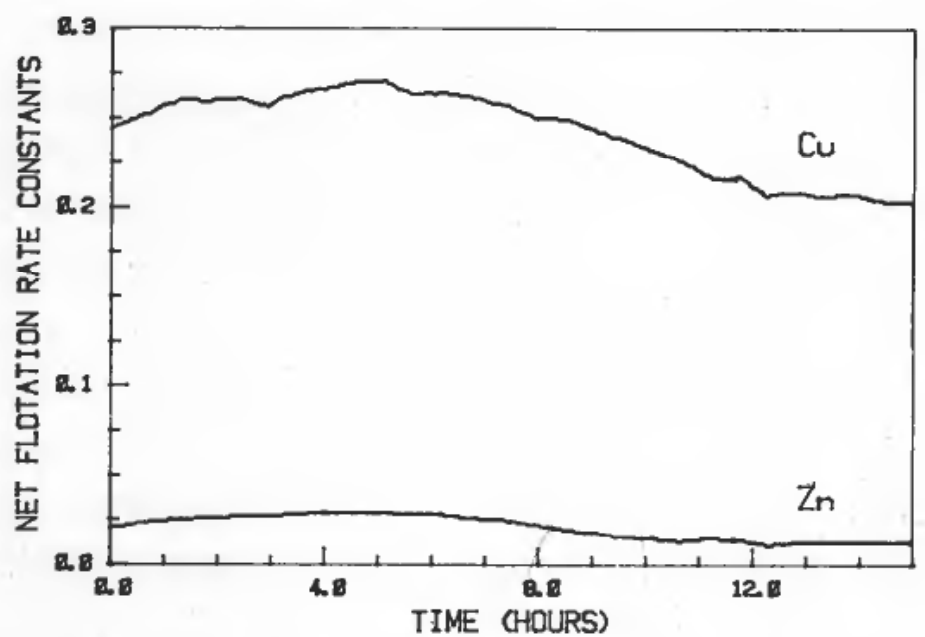

Figure 6. Estimated (and measured) grades and estimated aggregate net flotation rate constants of the first rougher bank at Folldal Verk, Norway. (a) Estimated and manually measured; (b) Estimated; (c) Estimated and measured; (d) Estimated.

$$
N_{j} \leftrightarrow \tilde{N}_{j}=\frac{1}{x_{p, j}^{N} \sum_{i=1}^{N} V_{p}^{i}}\left[\sum_{i=1}^{N}\left(V_{p}^{i} k_{p, j}{ }^{i} x_{p, j}^{i}-V_{s}^{i} k_{s, j}{ }^{i} x_{s, j}{ }^{i}\right)\right]
$$

flotation class $j=1, \ldots, n$

where $\sim$ denotes an aggregate variable (scalar or vector) in the multi-cell model, i.e., $\tilde{x}_{s}$ denotes the aggregate concentrate grades, $\tilde{k}_{p, j}, \tilde{k}_{s, j}$, and $\tilde{N}_{j}$ denotes, respectively, the aggregate flotation, drainage, and flotation rate constants for the $j$ th flotation class, whereas $\tilde{\gamma}$ actually is identical to $\gamma$ because $q_{p}{ }^{N}=q_{p}$ and $q_{p}{ }^{0}=q_{f}$.

Based on the foregoing aggregate model, an extended Kalman filter for the multicell process was developed. The form of this estimator is identical to the one which was developed for the single-cell process (Eqns. (15)-(20)). The observation equations have the form given by Eqns. (10)-(14). 


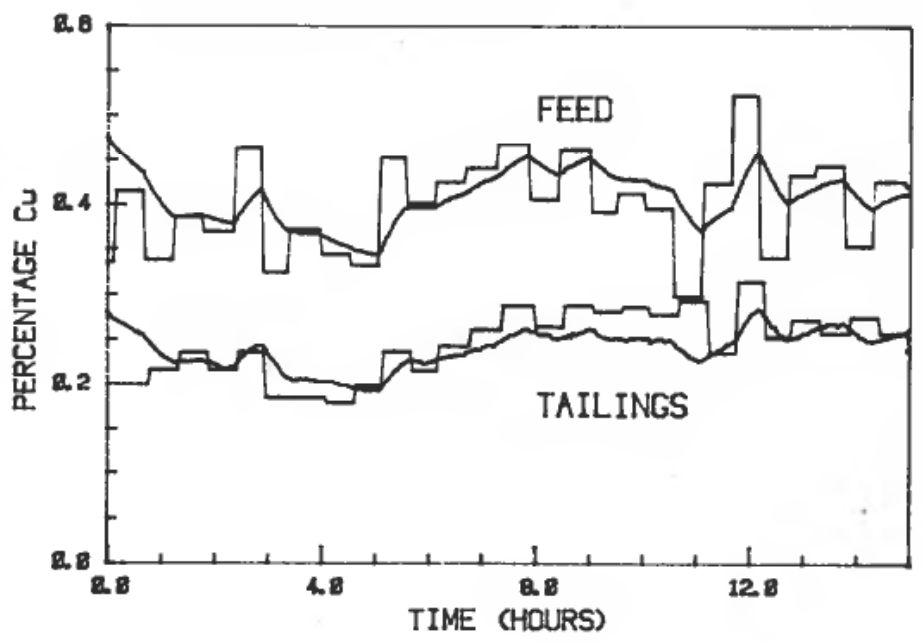

(c)

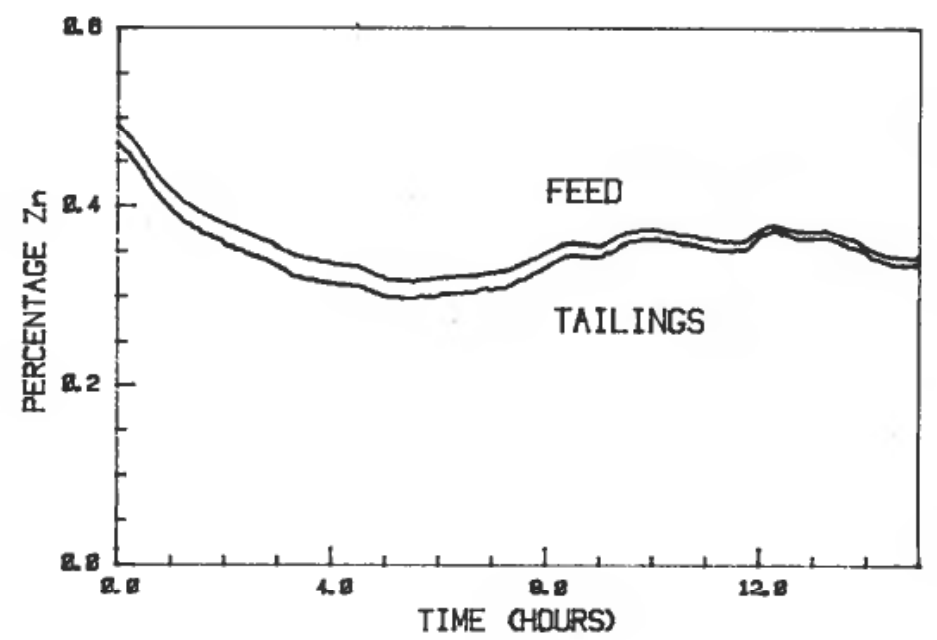

(d)

Figure 6-continued.

Typical samples from the simulation experiments with this estimator are shown in Fig. 5. The figure shows that the estimator is indeed able to estimate tailings grades, aggregate concentrate grades, and aggregate net flotation rate constants, whereas aggregate flotation and drainage rate constants could not be properly estimated (not shown in the figures). Actually, the simulation experiments revealed that the behaviour of the aggregate estimator was very similar to the behaviour of the estimator for a single-cell process, and they confirmed our belief that an aggregate estimator (based on a single-cell aggregate model) would be the most proper form of an estimator for a multi-cell process when only feed, tailings, and aggregate concentrate grades are being measured.

\section{Results from implementation in a real flotation plant}

The estimator which was developed in the previous section is part of an adaptive controller for the first rougher bank in the flotation plant at Folldal Verk, Norway. 
This is a four-cell process where a copper mineral (chalcopyrite) is separated from a zinc mineral (sphalerite) and gangue. One flotation class is used for each mineral, so that we actually have only two flotation classes. The number of state variables in the estimator is therefore equal to 9 (because drainage rate constants could be left out), whereas the number of states in the original (nonaggregated) model is 38 .

A very interesting point of the implementation was the fact that the concentrate grades could not be measured on-line. A seemingly essential measurement would therefore be missing, and we were quite uncertain about how the estimator would perform. On the other hand, this made the implementation of the estimator quite a bit more interesting since the concentrate grades could then only be measured by manual samples, a rather tedious and time-consuming task.

Recordings of the operation of the estimator made 20-21 November 1980 are shown in Figs. 6 and 7.

Figure $6(a)$ shows the estimated concentrate grades of copper and zinc. Also shown in this figure are the results from chemically analysed manual samples (black dots). The agreement between the estimator and the manual samples is quite good. It should be noted that the uncertainties in the manual samples are rather large, because the flow rate measurement are very uncertain.

It is obvious from Fig. $6(a)$ that the flotation process separated the two minerals quite well, and this fact is indeed supported by Fig. $6(b)$, which shows the aggregate net flotation rate constants of the two minerals. Figure $6(\mathrm{c})$ shows the estimated copper grades in the feed and the tailings together with the measured copper grades, i.e., the outputs from the X-ray analyser. The dynamic behaviour of the estimator is seen to be quite conservative due to the fact that the outputs from the X-ray analyser may be extremely uncertain. We can estimate the settling time from Fig. $6(c)$ to be approximately one hour. Figure $6(d)$ shows the estimated zinc grades in the feed and the tailings. They are, as shown, almost equal because very small amounts of zinc are actually being removed. Figure 7 shows the estimate of the parameter $\gamma$ plotted in a scale from 0.9 to $1 \cdot 0$. It seems to be rapidly changing, but plotted in a scale from 0.0 to $1 \cdot 0$, it virtually becomes a constant. The value of $1-\gamma$, however, varies significantly in relative terms.

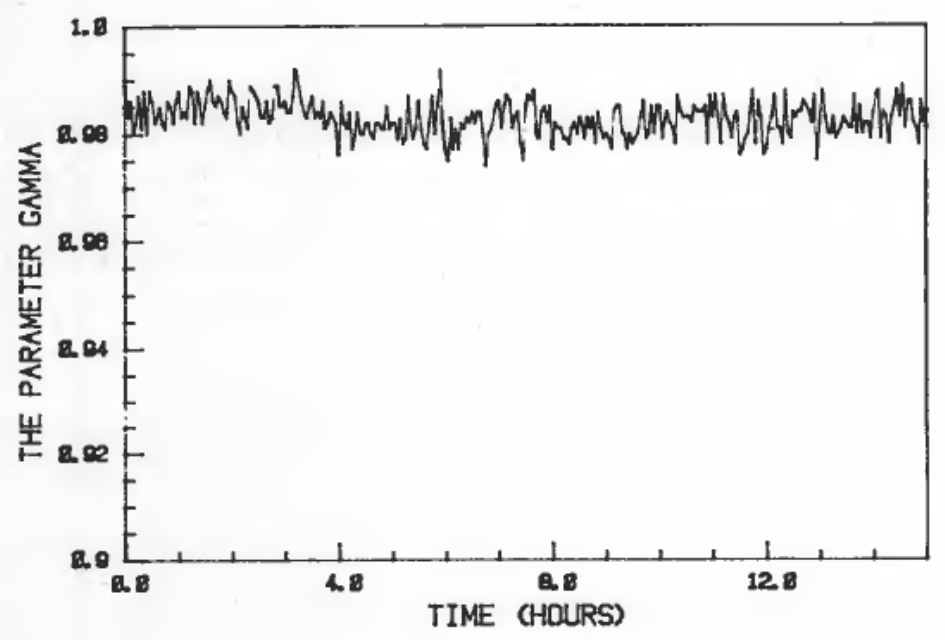

Figure 7. Estimate of the parameter $\gamma$ of the first rougher bank at Folldal Verk, Norway. 
Despite the fact that one seemingly essential observation (measurement of the concentrate grades), was not available in the actual implementation, the estimator worked well and gave satisfactory estimates of aggregate states and parameters in the process.

\section{Conclusion}

An on-line combined state and parameter estimator for a multi-cell flotation process has been developed through extensive simulation studies. The estimator is based upon an aggregated single-cell model of the plant because the simulation studies revealed that only aggregate states and net flotation rate constants could be properly estimated by measurements of the feed, the tailings, and, possibly, the concentrate grades. Results from the estimator's performance in a flotation plant show that it works satisfactory.

The reported estimator is part of an adaptive controller for the first rougher bank at Folldal Verk, Norway. Details about the design of this controller are given by Andersen et al. (1979). Some experiences from implementation and operation of the controller are reported by Kaggerud (1983).

\section{REFERENCES}

Andersen, R. W., Grønli, B., Olsen, T. O., KagGerud, I., Romslo, K., and Sandvik, K. L. (1979). An optimal control system of the rougher flotation at the Folldal Verk concentrator. Proceedings of the International Mineral Processing Congress, Warzaw, Poland, pp. 381-403.

Arbiter, N., and HARris, C. C. (1962). Flotation kinetics. In Froth Flotation (editor D. W. Fuerstenau) (AIME, New York), pp. 215-246.

Fournier, R. D., and Smith, H. W. (1972). On line estimation of flotation rates. Proceedings Ann. Meeting Amer. Inst. Chem. Eng., New York, N.Y., Paper No. 93-C.

FuerstenaU, M. C. (editor) (1976). Flotation (American Institute of Mining, Metallurgical and Petroleum Engineers, New York).

HAllingstad, O., and SALID, S. (1976). Calibration of a sea bottom moored transponder array. Proceedings IFAC/IFIP Symp. Automation in Offshore Oil Field Operation, Bergen, Norway, pp. 309-315.

Henriksen, R. (1982). The truncated second-order filter revisited. IEEE Trans. Automat. Control, AC-27, 247-251.

Henriksen, R., Kaggerud, I., and Olsen, T. O. (1982). Application of on-line estimation to a rougher flotation process. Proceedings 6th IFAC Symp. Identification and System Parameter Estimation, Arlington, Virginia, pp. 174-179.

JAZWinski, A. H. (1970). Stochastic Processes and Filtering Theory (Academic Press, New York).

KaGGerUD, I. (1983). Experiences with optimal control in a chalcopyrite flotation circuit. Proceedings 4th IFAC Symp. Automation in Mining, Mineral and Metal Processing, Espoo, Finland, pp. 427-433.

Kashyap, R. L. (1970). Maximum likelihood identification of stochastic linear systems. IEEE Trans. Automat. Control, AC-15, 25-34.

KING, R. P. (1975). Simulation of flotation plants. Society of Mining Engineers of AIME Trans., 258, 286-293.

MeHRA, R. K. (1971). Identification of stochastic linear dynamic systems using Kalman filter representation. AIAA Journal, 9, No. 1.

OlsEN, T. O. (1975). Mathematical models of flotation processes. The Norwegian Institute of Technology, Division of Engineering Cybernetics, Trondheim, Norway, Report No. 75-12-W. 
Olsen, T. O., and HeNRIKSEN, R. (1976). An innovations approach to parameter estimation in flotation processes. Proceedings 4th IFAC Symp. Identification and System Parameter Estimation, Tbilisi, USSR, Part 2, pp. 420-433.

Olsen, T. O., and HenRiksen, R. (1977). Comparison of different parameter estimation methods in flotation processes. Proceedings 4th IFAC Symp. Multivariable Technological Systems, Fredericton, Canada, pp. 463-468.

OLSEN, T. O., and HENRIKSEN, R. (1981). Identification of flotation processes using aggregated models Society of Mining Engineers of AIME Trans., 268, 1739-1744.

Sмгтн, H. W. (1975). Canadian experience in flotation control. Proceedings 6th IFAC World Congress, Boston/Cambridge, Mass., Paper No. 60.2.

VeTter, W. J. (1970). Derivative operations on matrices. IEEE Trans. Automat. Control, AC-15, 241-244.

VeTter, W. J. (1973). Matrix calculus operations and Taylor expansions. SIAM Rev., 15, 352-369. 